Check for updates

Cite this: J. Mater. Chem. B, 2020,

8, 5425

Received 12th April 2020

Accepted 27th May 2020

DOI: 10.1039/d0tb00962h

rsc.li/materials-b

\section{Plasmonic photothermal microneedle arrays and single needles for minimally-invasive deep in-skin hyperthermia $\dagger$}

\author{
Álvaro Cárcamo-Martínez, ${ }^{a}$ Brónach Mallon, ${ }^{a}$ Juan Domínguez-Robles, ${ }^{a}$ \\ A. Sara Cordeiro, (D) ${ }^{a}$ Maurizio Celentano, (D) ${ }^{b}$ Eneko Larrañeta, ${ }^{a}$ Steven E. J. Bell (iD ${ }^{b}$ \\ and Ryan F. Donnelly (D) *a
}

\begin{abstract}
We report, for the first time, crosslinked polymeric microneedle (MN) arrays and single needles ( $2 \mathrm{~mm}$ and $4.5 \mathrm{~mm}$ length) coated with gold nanorods (GnRs) to induce deep hyperthermia in a $3 \mathrm{~mm}$ thickness skin model upon near infrared (NIR) laser irradiation. Using excised neonatal porcine skin as tissue model, it was seen that insertion capabilities of single prototypes were not affected by the coating, as around $\mathbf{8 0} \%$ of their length was inserted before and after coating. Insertion of $\mathrm{MN}$ arrays dropped from $74 \%$ to $55 \%$, which could be attributed to a less sharp structure after the coating process. Nonetheless, GnRs-coated MN arrays achieved the highest increase in temperature in the skin model: over $15^{\circ} \mathrm{C}$ after only $15 \mathrm{~s}$ of NIR laser irradiation $(808 \mathrm{~nm}$, $2 \mathrm{~W} \mathrm{~cm}^{-2}$ ). Surprisingly, removal of $\mathrm{MN}$ arrays after irradiation left no detectable polymer or plasmonic material behind, confirming the enhanced safety and minimally-invasive potential of this device for future biomedical applications of deep in skin hyperthermia.
\end{abstract}

\section{Introduction}

The use of hyperthermia mediated by gold nanorods (GnRs) for biomedical applications has been extensively reported in the literature as upon near-infrared (NIR) irradiation, GnRs can emit heat by absorbing this irradiation on the basis of the phenomenon of localized surface plasmon resonance (LSPR). ${ }^{1}$ Since a temperature increase in cells can elicit immune and cytotoxic responses, hyperthermia can be useful for treatment of a wide variety of conditions, ranging from bacterial skin infections to non-melanoma skin cancers (NMSC). ${ }^{2-5}$ Indeed, gold nanorods have been explored as primary and adjunctive therapy for photothermal ablation of acne-responsible bacteria, ${ }^{6}$ subcutaneous abscesses and tissue infections. ${ }^{7,8}$ NMSC murine models have also been targeted with GnRs, where particles are injected intratumorally or systemically. ${ }^{9-12}$

\footnotetext{
${ }^{a}$ School of Pharmacy, Queen's University Belfast, Belfast BT9 7BL, UK.

E-mail: R.Donnely@qub.ac.uk

${ }^{b}$ School of Chemistry and Chemical Engineering, Queen's University Belfast, Belfast BT9 5AG, UK

$\dagger$ Electronic supplementary information (ESI) available. See DOI: 10.1039/d0tb00962h
}

Less invasive approaches have focused on loading these particles into hydrogels, ${ }^{13,14}$ composites ${ }^{15,16}$ and microneedle (MN) arrays $^{17-19}$ to explore GnRs-mediated superficial skin hyperthermia. Although effective, all of these approaches have not reported toxicity of GnRs once they are deposited into the skin and/or reach the systemic circulation. Surface chemistry, size and concentration of GnRs have been proposed as the factors responsible for cell toxicity in in vitro studies (using dermal fibroblasts and HaCaT cells among others) ${ }^{20-23}$ and in vivo studies in zebrafish ${ }^{24,25}$ and mice. ${ }^{20,26}$ Hence, research should focus now on exploiting plasmonic properties of GnRs but avoiding skin deposition or injection routes.

In our previous work, GnRs were included in polymeric films made from aqueous blends of Gantrez ${ }^{\mathbb{R}}$ S-97 (a copolymer of methyl vinyl ether and maleic acid (PVME/MA) and poly(ethylene glycol) (PEG) $200 \mathrm{Da}$, aiming to induce superficial hyperthermia upon NIR laser irradiation. ${ }^{27}$ Films were able to heat a piece of excised neonatal porcine skin ( $700 \mu \mathrm{m}$ thickness) up to around $40{ }^{\circ} \mathrm{C}$. Importantly, GnRs were trapped in the crosslinked polymeric network, as shown in release studies, even after immersing the films in PBS for one hour. Hence, this technology circumvents skin deposition and injection of GnRs and, thereby, the uncertainty regarding their metabolism, safety and toxicity once they reach the systemic circulation. To improve this system and expand its potential use for deep in skin hyperthermia, a proof of concept study is reported herein, where hydrogel-forming microneedle $(\mathrm{MN})$ arrays $(5 \times 5$ needles, $1 \mathrm{~mm}$ in length $)$ and two types of single needles ( $2 \mathrm{~mm}$ and $4.5 \mathrm{~mm}$ length) coated with GnRs were fabricated. These plasmonic prototypes were tested in terms of resistance to compression and insertion capabilities, comparing also their ability to increase the temperature of a $3 \mathrm{~mm}$-thick skin model upon laser irradiation.

\section{Materials}

Gantrez ${ }^{\circledR}$ S-97, a copolymer of methyl vinyl ether and maleic acid (PVME/MA), with a molar mass of 1200000 was a donated 
from Ashland (Kidderminster, UK). Tetrachloroauric acid $\left(\mathrm{HAuCl}_{4}\right)$, cetyltrimethylammonium bromide (CTAB), sodium borohydride $\left(\mathrm{NaBH}_{4}\right)$, L-ascorbic acid, and poly(ethyleneglycol) (PEG) $200 \mathrm{Da}$ were purchased from Sigma-Aldrich (Missouri, USA). 5-Bromosalicylic acid (5-BrSA) and silver nitrate $\left(\mathrm{AgNO}_{3}\right)$ were purchased from Alfa Aesar (Lancashire, UK). IP-S photoresin was purchased from Nanoscribe (Eggenstein-Leopoldshafen, Germany). Poly(lactic acid) (PLA) was purchased from Ultimaker B. V. (Geldermalsen, The Netherlands). Poly(dimethylsiloxane) (PDMS), silicone elastomer, was purchased from Polymer Systems Technology (High Wycombe, UK). Silastic ${ }^{\circledR}$ S, silicone rubber and curing agent, was purchased from Thompson Bros. Ltd. (Newcastle Upon Tyne, UK). All other chemicals were of analytical grade. Additionally, two types of excised porcine skin were used. For insertion studies, skin with underlying connective tissue $(3.5 \mathrm{~cm}$ thick) was obtained from a local supplier. For heating studies, full thickness neonatal porcine skin (1.5 mm thick) was obtained from still-born piglets. In both cases the skin was rinsed in PBS and frozen at $-20{ }^{\circ} \mathrm{C}$ prior to use.

\section{Methods}

\section{Synthesis and characterisation of GnRs}

GnRs were prepared by a seed-mediated process previously reported. ${ }^{28}$ To determine their absorbance properties, a UV microplate FluoStar Optima fluorescence spectrophotometer (BMG Labtech, Ortenberg, Germany) was used. Transmission electron microscopy (TEM) was used to measure the length and width of GnRs. For this purpose, $10 \mu \mathrm{L}$ of washed samples were deposited on copper grids and evaluated using a JEM-1400Plus microscope (JEOL, Tokyo, Japan). A NanoBrook Omni particle sizer and zeta potential analyzer (Brookhaven, New York, NY, USA) was used to measure the zeta potential of GnRs. Gold content in GnRs samples was assessed by inductively coupled plasma optical emission spectroscopy (ICP-OES) using a 5100 Synchronous Vertical Dual View ICP (Agilent, California, USA). Accordingly, GnRs samples of $50 \mathrm{~mL}$ were centrifuged at $8500 \mathrm{rpm}$ for $45 \mathrm{~min}$, the precipitate was resuspended in purified water and centrifuged again. Then, aliquots of $2.5 \mathrm{~mL}$ of GnRs were mixed with nitric acid $65 \%$, hydrochloric acid $37 \%$ and purified water to have a final volume of $50 \mathrm{~mL}$. The solution was kept at $60{ }^{\circ} \mathrm{C}$ for 2 hours to digest and dissolve all materials and then the gold content was measured.

\section{Fabrication and characterisation of GnRs-coated prototypes}

Polymeric single needles and MN arrays were fabricated by a micro-moulding technique, using silicone moulds previously fabricated. Briefly, MN arrays master templates were printed using a Nanoscribe 3D printer (Nanoscribe GmbH, Germany), which is based and IP-S resin as printing material. Master templates consisted of an array of $5 \times 5 \mathrm{MNs}, 1.00 \mathrm{~mm}$ length each. Once printed, master templates were attached to a $3 \mathrm{D}$ printed holder (Ultimaker3Printer) with cyanoacrylate glue. PDMS was cast into the holders, centrifuged at $3500 \mathrm{rpm}$ for $10 \mathrm{~min}$ and cured for 4 hours at $80{ }^{\circ} \mathrm{C}$ to obtain silicon moulds. Single needles master moulds were prepared as previously reported, ${ }^{29}$ using a 3D printer Ultimaker 3 (Ultimaker B.V., Geldermalsen, The Netherlands) and PLA as printing material to fabricate the holder. Then, metallic needles were inserted and glued to these bases using cyanoacrylate. A mixture of Silastic $^{\mathbb{R}} \mathrm{S}$ and a curing agent, in a proportion of $10: 1$, was poured into the master moulds and allowed to cure overnight to obtain silicone moulds. Dimensions of single needles moulds were $2 \mathrm{~mm}$ length $/ 464 \mu \mathrm{m}$ base width and $4.5 \mathrm{~mm}$ length/922 $\mu \mathrm{m}$ base width. The preparation of GnRs-coated prototypes is shown in Fig. 1. A hydrogel (w/w\%) made from aqueous blends containing 25\% Gantrez ${ }^{\circledR}$ S-97 and 10\% PEG 200 Da was poured into silicone moulds. Single needle moulds were centrifuged at $5000 \mathrm{rpm}$ for $20 \mathrm{~min}$, whereas MN array moulds were placed in a pressure chamber at 5 bar for $15 \mathrm{~min}$. Then, needles were dried at room temperature for 2 days and placed after in an oven at $80{ }^{\circ} \mathrm{C}$ overnight to induce ester-based crosslinking between PEG and Gantrez ${ }^{\circledR}$ S-97. Afterwards, a coating mixture $(\mathrm{w} / \mathrm{w} \%)$ containing $5 \%$ Gantrez ${ }^{\circledR}$ S-97, 2.5\% PEG $200 \mathrm{Da}, 2.5 \%$ Tween $^{\circledR} 20,22.5 \%$ purified water and q.s. GnRs suspension was prepared. The coating was performed by dropping $70 \mu \mathrm{L}$ of the coating mixture on top of the needles and

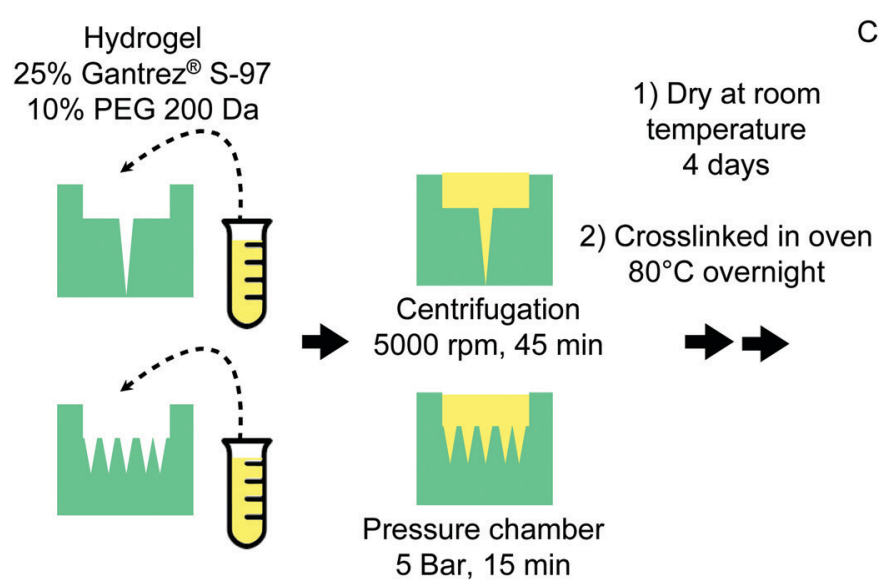

Coating formulation $70 \mu \mathrm{L}$

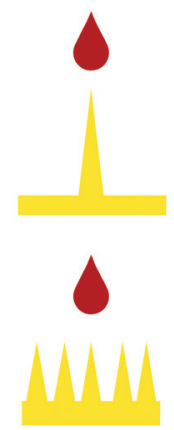

Crosslinked in oven $80^{\circ} \mathrm{C}$ overnight

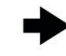

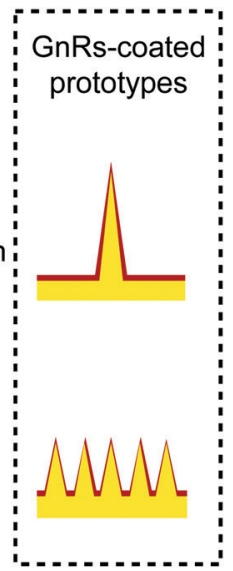

Fig. 1 Schematic representation of the fabrication of GnRs-coated prototypes. 
crosslinked immediately in an oven at $80{ }^{\circ} \mathrm{C}$ overnight. A light microscope Leica EZ4 D stereo (Leica Microsystems, Milton Keynes, UK) and a Tabletop TM3030 scanning electron microscope (Hitachi, Tokyo, Japan) were used to visualise prototypes dimensions. GnRs-coated length was assessed using light microscopy due to the difference in colours between hydrogelforming needles and GnRs-coating formulation.

\section{Resistance to compression and insertion capabilities of GnRs-coated prototypes}

Effect of GnRs-coating on resistance to compression of prototypes was tested with a Texture Analyser TA-XT2 (Stable Microsystems, Haslemere, UK) set in compression mode (pre-test speed: $1 \mathrm{~mm} \mathrm{~s}^{-1}$, test speed: $1 \mathrm{~mm} \mathrm{~s}^{-1}$ and post-test speed: $1 \mathrm{~mm} \mathrm{~s}^{-1}$ ) and using a force of $32 \mathrm{~N}$ per $30 \mathrm{~s}$ as the target mode. ${ }^{30}$ Prototypes were attached with double-sided tape to the mobile probe and compressed against a flat metallic surface on the base of the equipment. The length of the needles before and after compression was recorded using light microscopy and the percentage of height reduction was calculated. Similarly, insertion capability of prototypes was tested by attaching them to the mobile probe of the Texture Analyser and the skin model was placed on the metallic base using the same compression mode and parameters above-mentioned. Due to the length of single needles, porcine skin with underlying connective tissue was used on this case. Once needles were inserted, optical coherence tomography (OCT), EX1201 OCT Microscope (Michelson Diagnostics Ltd, Kent, UK), was used to measure the percentage of needle's height effectively inserted, employing a known correlation between pixels and $\mu \mathrm{m}$ (1 pixel equals to $4.2 \mu \mathrm{m})$, as previously reported. ${ }^{30,31}$

\section{Thermal studies of GnRs-coated prototypes}

The thermal capacity of GnRs-coated prototypes was evaluated using a $2 \mathrm{~W} \mathrm{~cm}^{-2} 808 \mathrm{~nm}$ infrared laser (Sunshine-Electronics,
Guangdong, China) and an infrared thermal imaging camera FLIR A35sc (FLIR, Täby, Sweden), as shown in Fig. 2A. Prototypes were placed at a distance of $17 \mathrm{~cm}$ from the laser, attaching them with double-sided tape to a plastic hollow holder. The thermal camera was placed at a distance of $2 \mathrm{~cm}$ from GnRs-coated needles. Temperature of each prototype was recorded before and after NIR irradiation for $10 \mathrm{~s}$. In all cases, prototypes without GnRs-coating were used as controls.

\section{Heating capacity of GnRs-coated prototypes on a skin model}

To determine whether the coated prototypes would have the capacity to transfer the generated heat upon laser irradiation to a skin model, the set up shown in Fig. 2B was used. Two pieces of full thickness neonatal porcine skin, having a total thickness of $3 \mathrm{~mm}$, were placed facing each other's dermis and pinned down on a poly(styrene) foam board with a square hole in the centre. The laser was positioned at $17 \mathrm{~cm}$ from the upper side of the skin where prototypes were inserted. In order to evaluate the heating in the skin model, the thermal imaging camera was placed $2 \mathrm{~cm}$ under the poly(styrene) foam, focusing at the side of the skin visible due to the square hole (underside view). GnRs-coated prototypes were manually inserted into the skin, pressed for $30 \mathrm{~s}$ and irradiated for $15 \mathrm{~s}$. Temperature before and after irradiation was recorded.

\section{Statistical analyses}

Data is shown as means \pm standard deviation (SD) from triplicate measurements, unless otherwise stated. Differences between study groups were assessed for significance using one-way analysis of variance (ANOVA), followed by a multiple comparisons test (Tukey's test) or with a $t$-test (for 2 groups). In both cases, the threshold for significance was $p<0.05$. Statistical analysis were performed using GraphPad Prism ${ }^{\circledR}$ version 7 (GraphPad Software, San Diego, CA, USA).
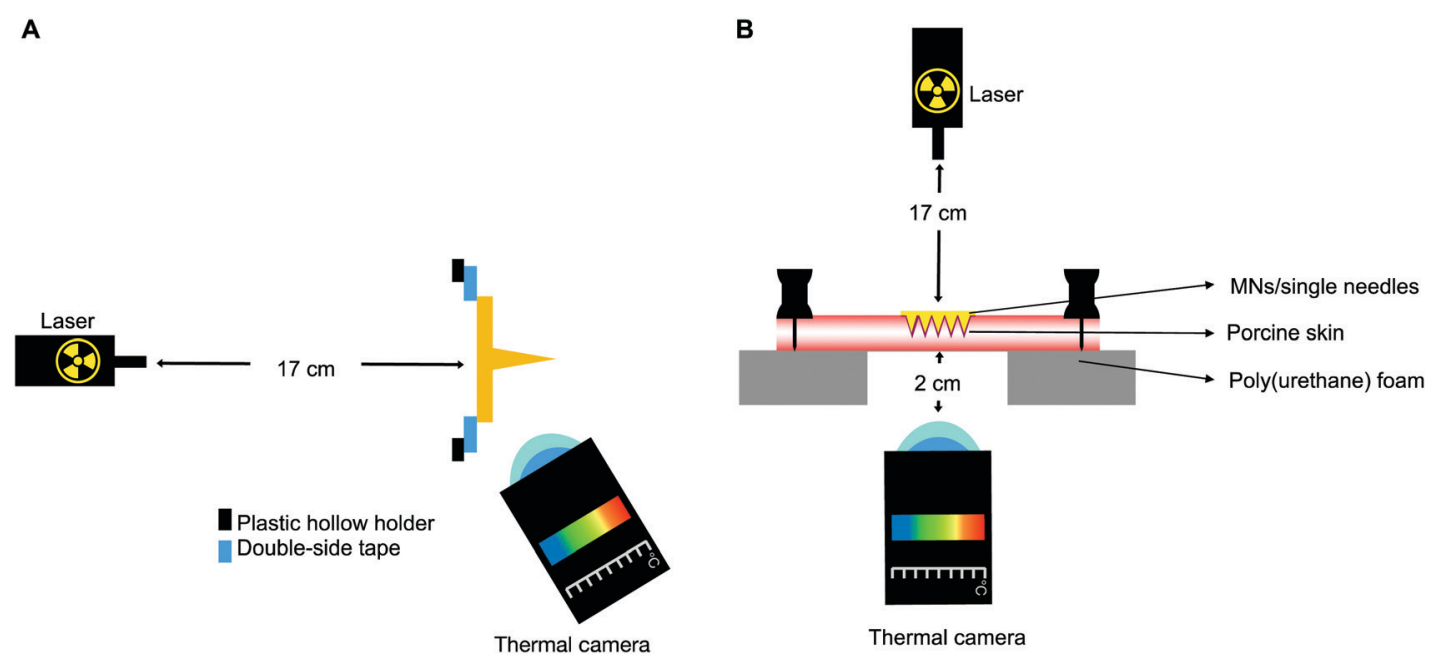

Fig. 2 Experimental set-up for thermal studies of GnRs-coated prototypes (A). Distance between laser and needles was kept at $17 \mathrm{~cm}$. Laser wavelength was $808 \mathrm{~nm}$ with a power of $2 \mathrm{~W} \mathrm{~cm}^{-2}$ and needles irradiation lasted for $10 \mathrm{~s}$. Experimental set-up for heating capacity of GnRs-coated prototypes on a skin model (B). Full-thickness neonatal porcine skin (2 pieces facing each other's dermis, $3 \mathrm{~mm}$ total thickness) were pinned down on a poly(styrene) foam. Laser wavelength was $808 \mathrm{~nm}$ with a power of $2 \mathrm{~W} \mathrm{~cm}^{-2}$ and prototypes were irradiated for $15 \mathrm{~s}$. 


\section{Results}

\section{Synthesis and characterisation of GnRs}

GnRs were prepared using a seed-mediated process, achieving a rod-like shape, as shown in Fig. 3. Length, width and aspect ratio of GnRs were $58.54 \pm 5.31 \mathrm{~nm}, 19.95 \pm 0.91 \mathrm{~nm}$ and $2.94 \pm 0.33$, respectively (mean \pm S.D., $n=5$ ). Maximum longitudinal absorbance peak was at $767 \mathrm{~nm}, z$-potential was $27.87 \pm 6.15 \mathrm{mV}$ (mean \pm S.D., $n=9$ ) and ICP-OES measurements indicated that $7.95 \mu \mathrm{g} \mathrm{mL}^{-1}$ of gold were present in the GnRs suspension.

To achieve optimal tissue hyperthermia, GnRs with high photon-to-heat conversion efficiency are required, which is maximized by using small rods, like the ones here fabricated. GnRs with higher dimensions have been reported as ideal for biolabeling and contrast agent purposes as, in such cases, light scattering instead of absorption becomes dominant. ${ }^{32}$

\section{Fabrication and characterisation of GnRs-coated prototypes}

To minimise possible release of GnRs during insertion, irradiation and removal of the plasmonic devices from the skin, a hydrogel formulation containing a crosslinking agent with low molecular weight (PEG $200 \mathrm{Da}$ ) was developed to coat the prototypes. Swelling studies of formulations containing Gantrez ${ }^{\circledR}$ S-97 and PEG have been already reported in literature, where the highly dependent effect of the molecular weight of the crosslinking agent used on the swelling capacity of hydrogels is described. ${ }^{33}$

The inclusion of PEG 200 Da led to a hydrogel with rigid networks and high crosslink densities. Hence, inclusion of GnRs in a formulation like this could lead to low swelling rates, helping to retain GnRs in the crosslinked network. Furthermore, Tween ${ }^{\circledR} 20$ was added into the formulation to decrease surface tension as this has been reported as a crucial parameter to achieve a uniform spread of coatings on $\mathrm{MN}$ arrays. ${ }^{34}$ Representative images of coated prototypes and physical characterisation data are shown in Fig. 3 and Table 1, respectively.

A full coating was achieved in $\mathrm{MN}$ arrays, whereas only part of the total length of single needles was coated. Other strategies were tested to increase the coated length of single needles, as shown in Fig. S1 (ESI $\dagger$ ), but none of them led to improved results. Nonetheless, a uniform film can be seen in all needles with a smooth surface and no bubbles or cracks. From the images, it can be also seen that MN arrays were less sharp after coating and single needles increased their width.

\section{Resistance to compression and insertion capabilities of GnRs-coated prototypes}

To compare the effect of coating on the resistance to compression of prototypes, a Texture Analyser was used and the length of needles before and after the application of a $32 \mathrm{~N}$ axial force was measured, as shown in Fig. 4A. Single needle of $2 \mathrm{~mm}$ length did not experience any significant reduction in height. However, $4.5 \mathrm{~mm}$ single needles and MN arrays did indeed show significant differences, which could be attributed to the clear increase on base width after being coated $(p<0.05)$. Afterwards, insertion of prototypes was tested using excised porcine skin with underlying connective tissue (around $3.5 \mathrm{~cm}$ in depth), inserting all needles manually for $30 \mathrm{~s}$ (Fig. 4B). Both single-needle prototypes (2 and $4.5 \mathrm{~mm}$-length needles) inserted around $80 \%$ of their total length on the skin model, showing no differences before and after coating $(p>0.05)$.

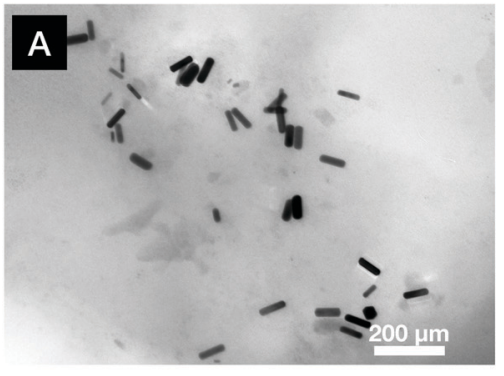

B

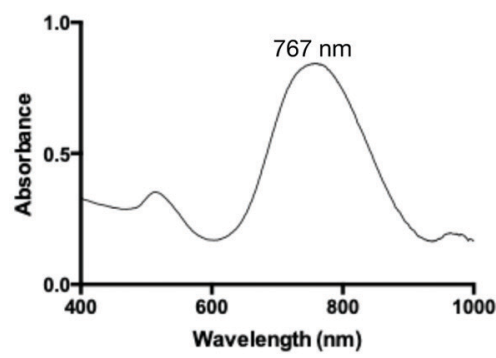

$5 \times 5,1 \mathrm{~mm}$

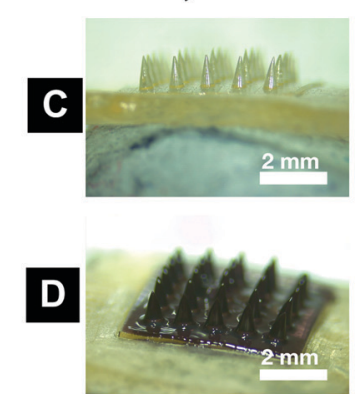

$\mathbf{E}$
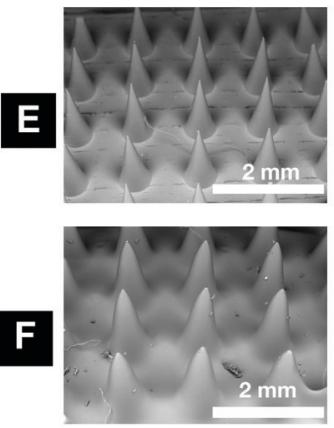

$2 \mathrm{~mm}$
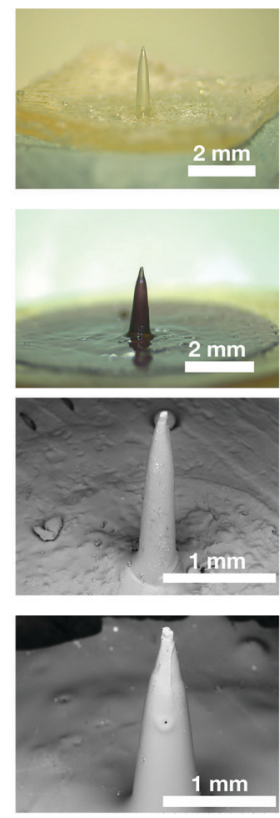

$4.5 \mathrm{~mm}$
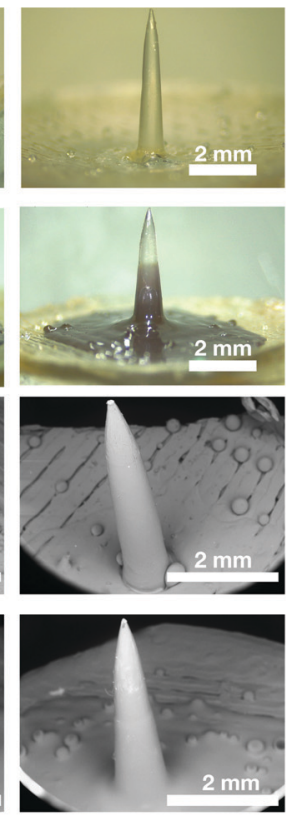

Fig. 3 TEM images of GnRs (A) and UV-vis absorbance spectrum of GnRs (B). Representative light microscopy images of prototypes before (C) and after coating (D). Scanning electron microscopy of prototypes before (E) and after coating (F). 
Table 1 Physical properties of GnRs-coated prototypes (means \pm SD, $n=3$ )

\begin{tabular}{|c|c|c|c|c|c|c|c|}
\hline \multirow[b]{2}{*}{ Prototype } & \multicolumn{3}{|l|}{ Before coating } & \multicolumn{4}{|l|}{ After coating } \\
\hline & Length (mm) & Width at base $(\mathrm{mm})$ & Aspect ratio & Length (mm) & Width at base (mm) & Aspect ratio & Length coated (\%) \\
\hline MN arrays & $1.05 \pm 0.05$ & $0.48 \pm 0.01$ & $2.20 \pm 0.11$ & $1.03 \pm 0.05$ & $0.63 \pm 0.01$ & $1.63 \pm 0.08$ & 100 \\
\hline $2 \mathrm{~mm}$ needle & $2.10 \pm 0.04$ & $0.49 \pm 0.02$ & $4.33 \pm 0.24$ & $2.21 \pm 0.09$ & $0.69 \pm 0.02$ & $3.23 \pm 0.07$ & $73.38+6.34$ \\
\hline $4.5 \mathrm{~mm}$ needle & $4.52 \pm 0.06$ & $0.93 \pm 0.05$ & $4.87 \pm 0.24$ & $4.55 \pm 0.11$ & $1.23 \pm 0.03$ & $3.70 \pm 0.03$ & $52.19+2.40$ \\
\hline
\end{tabular}

A

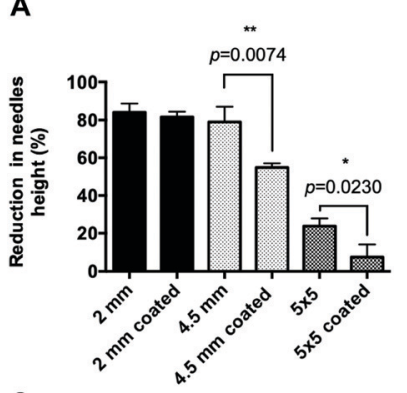

B

C
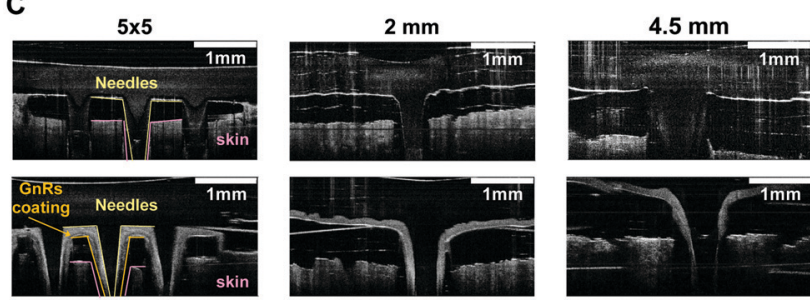

Fig. 4 Reduction in height of prototypes after a $32 \mathrm{~N}$ axial force was exerted on them (means $+\mathrm{SD}, n=3$ ) (A). Insertion depth of prototypes before and after coating on porcine skin (means $+\mathrm{SD}, n=3$ ) (B). Representative OCT images of prototypes insertion on porcine skin before and after coating (C).

On the other hand, coated MN arrays decreased their insertion capabilities significantly ( $p=0.0230$ ), dropping from $74 \%$ to $55 \%$ of inserted length, which could be a consequence of a less sharp structure after coating. In addition, representative images of prototypes insertion into the skin model using OCT can be seen in Fig. 4C. GnRs absorb light in the NIR region, but they also can reflect it. ${ }^{35}$ Since OCT operates with a NIR laser and particles are highly concentrated in the coating, GnRs and the hydrogel that forms the needles can be clearly differentiated with this technique.

\section{Thermal studies of GnRs-coated prototypes}

Prototypes were evaluated in terms of heating capability by irradiating them with a NIR laser for $10 \mathrm{~s}\left(808 \mathrm{~nm}\right.$ and $2 \mathrm{~W} \mathrm{~cm}^{-2}$, placed at $17 \mathrm{~cm}$ from the needles) and the increase in temperature recorded with a thermal imaging camera placed at $2 \mathrm{~cm}$ from prototypes. Representative images of the irradiated GnRs-coated prototypes and the obtained values for the increase in temperature are shown in Fig. 5. A clear and significant increase in temperature can be seen when needles were coated with GnRs (in all comparisons $p<0.05$ ). Temperature increase for single needles was measured by recording this increase on 3 different points: base, middle and tip of the needles. In the case of $\mathrm{MN}$ arrays, and due to their smaller dimensions, only the temperature increase of the central needle was recorded. It can be seen that all non-coated needles increased their temperature by less than $1{ }^{\circ} \mathrm{C}$. Furthermore, in the case of $2 \mathrm{~mm}$ GnRs-coated needles (Fig. 5G), an increase in temperature of 35,65 and $91{ }^{\circ} \mathrm{C}$ was seen in tip, middle and base, respectively. A similar trend was observed in $4.5 \mathrm{~mm}$ needles with values of 4.6, 15.1 and $44.7^{\circ} \mathrm{C}$ from tip to base. Finally, MN arrays increased in temperature to $57.2^{\circ} \mathrm{C}$ after $10 \mathrm{~s}$ of irradiation. Differences in temperature in single needles can be explained by a higher concentration of GnRs towards the base of the needles, as the coating formulation was dropped at the needles' tip, and then spread towards the baseplate. Under the same rational, it was then expected that $2 \mathrm{~mm}$ needle reached a higher temperature than the $4.5 \mathrm{~mm}$ needles and the MN arrays, since the same amount of GnRs had been spread over a smaller surface.

\section{Heating capacity of GnRs-coated prototypes on a skin model}

To determine whether the coated prototypes would have the capacity to transfer the generated heat upon laser irradiation to a skin model, two pieces of neonatal full thickness porcine skin facing each other's dermis, having a total thickness of $3 \mathrm{~mm}$, were used.

The same thermal imaging camera was placed underneath the porcine skin to record the underside increase of temperature, placed $2 \mathrm{~cm}$ from the skin. GnRs-coated prototypes were manually inserted, pressed for $30 \mathrm{~s}$ and initial temperature of the skin model was recorded. Prototypes were then irradiated for $15 \mathrm{~s}$ ( $808 \mathrm{~nm}$ and $2 \mathrm{~W} \mathrm{~cm}^{-2}$ laser, placed at $17 \mathrm{~cm}$ from the needles), recording the temperature reached. Representative images of MN array-based heating of the skin model are shown in Fig. 6A, where control represents direct irradiation of neonatal porcine skin without MN arrays or single needles inserted. Temperature increase was measured by recording the maximum temperature reached, as shown in Fig. 6B, where clear differences can be seen between the different prototypes. Prototypes of $4.5 \mathrm{~mm}$ length, $2 \mathrm{~mm}$ length and $5 \times 5 \mathrm{MN}$ arrays achieved a maximum increase of temperature in the skin model of $6.77,10.1$ and $15.95{ }^{\circ} \mathrm{C}$, respectively. Such differences could be the result of several factors. Even though single prototypes had a higher insertion in porcine skin, MN arrays have a higher density of needles penetrating the skin as well as the baseplate in closer contact with it. Hence, this may have contributed to this higher temperature increase. To further compare the heating capacity of the different prototypes on neonatal porcine skin, the area under the curve (AUC) was calculated and compared (Fig. 6C), where it can be clearly seen that $\mathrm{MN}$ arrays were the prototype that heated the skin model most significantly $(p<0.0001)$. 

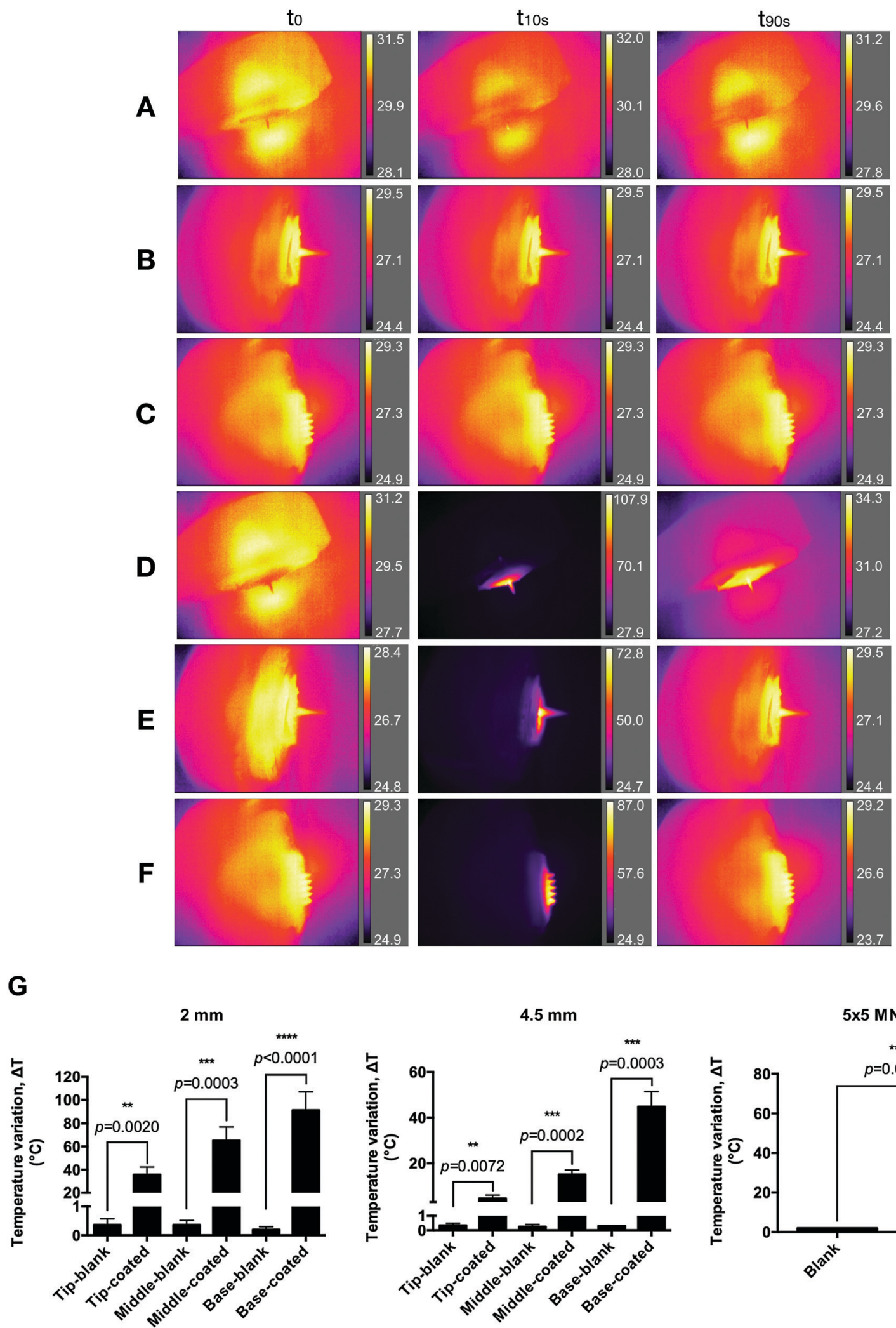

$4.5 \mathrm{~mm}$

$5 \times 5 \mathrm{MN}$ array
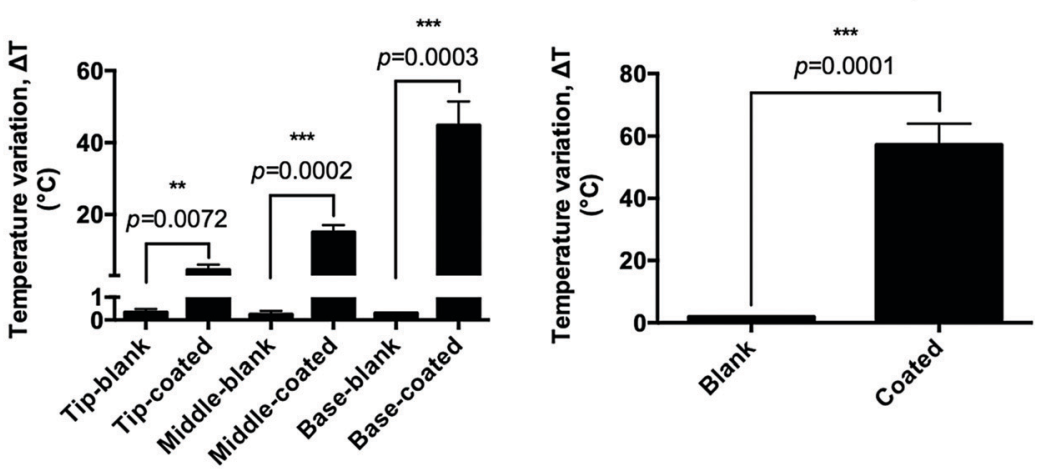

Fig. 5 Representative thermal images of prototypes before and after irradiation. Non-coated $2 \mathrm{~mm}$ length needle (A), non-coated $4.5 \mathrm{~mm}$ length needle (B), non-coated MN array (C), coated $2 \mathrm{~mm}$ length needle (D), coated $4.5 \mathrm{~mm}$ length needle (E), coated MN array (F). Temperature increase of: $2 \mathrm{~mm}$ length, $4.5 \mathrm{~mm}$-length needles and $5 \times 5$ arrays (G). Each prototype was irradiated for $10 \mathrm{~s}$ (means $+\mathrm{SD}, n=3$ ). 
A

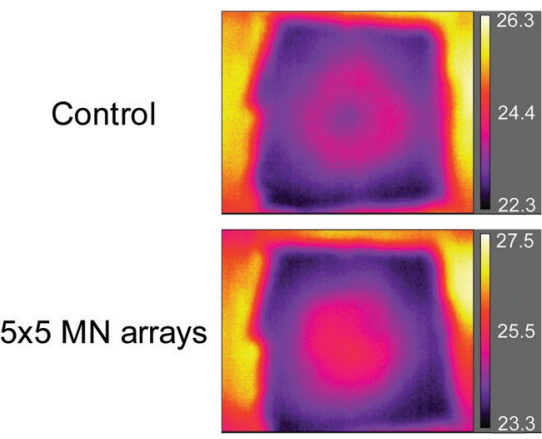

B

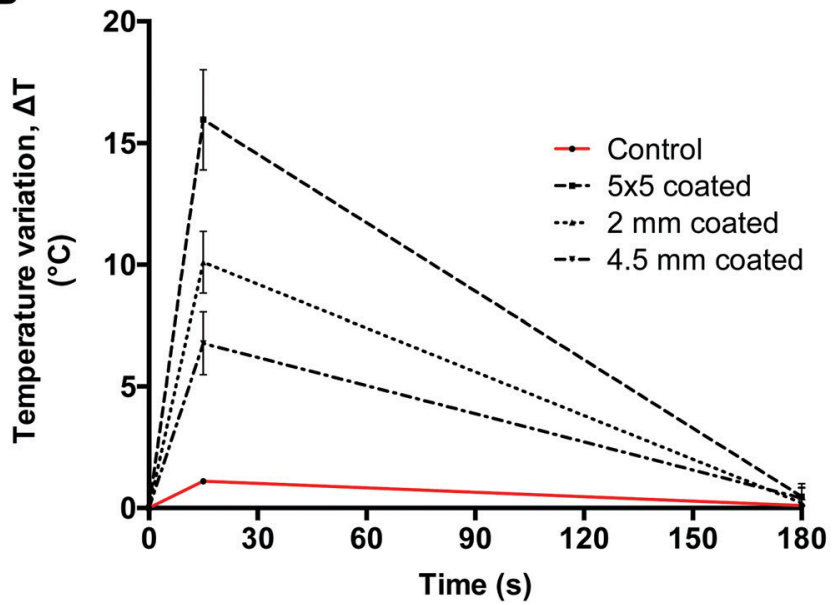

$\mathrm{t} 15 \mathrm{~s}$
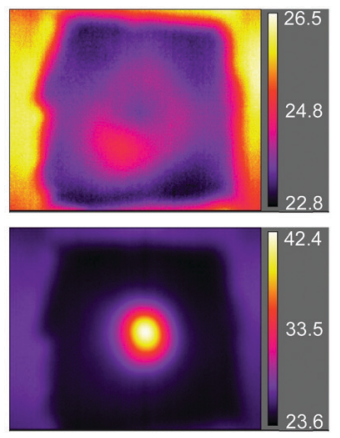

C t180s
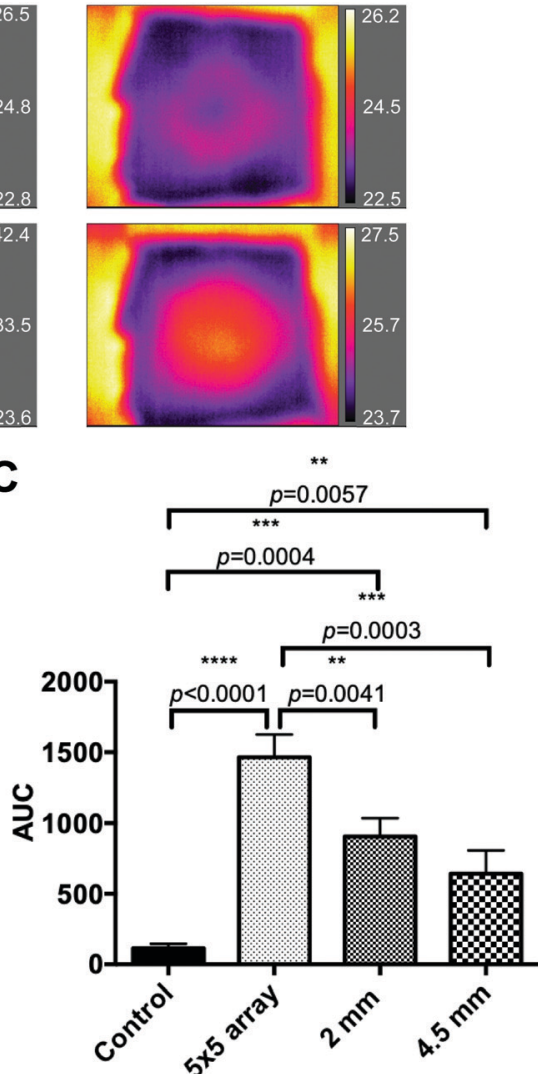

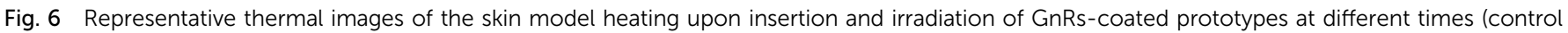

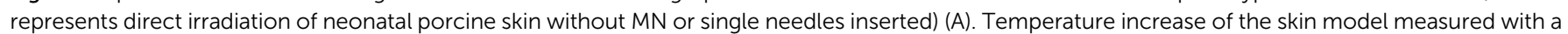
thermal camera (means $\pm \mathrm{SD}, n=3$ ) (B). AUC of skin model heating using plasmonic prototypes (means $+\mathrm{SD}, n=3)(\mathrm{C})$.

After insertion of GnRs-coated MN arrays into neonatal porcine skin, irradiation and removal, images of the prototype were taken, as shown in Fig. 7. After removal, the GnRs coating slightly detached from the MN arrays (Fig. 7A), which was
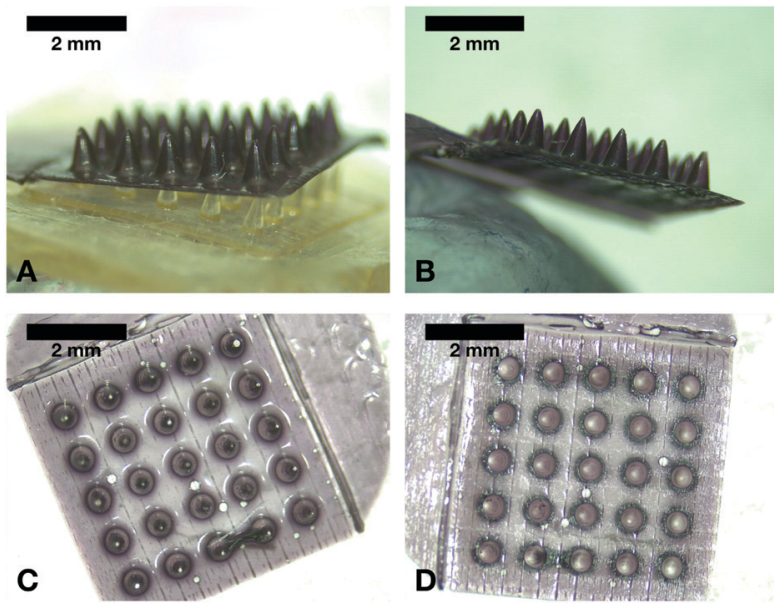

Fig. 7 Representative light microscopy images of $5 \times 5$ GnRs-coated MN arrays after insertion into neonatal porcine skin. The coating peels off after needles removal from the skin model ( $A$ and $B$ ) and integrally as shown in a top (C) and underside (D) view. afterwards manually peeled off (Fig. 7B-D). By doing so, it was noticed that the coating could be fully separated and came off on a single piece. This showcases the enhanced safety of GnRs-coated MN arrays since any chances of skin deposition of the polymeric and plasmonic materials are minimised.

Current treatments for NMSC lesions involve photodynamic therapy (PDT) and surgery. ${ }^{36}$ Unfortunately, poor skin penetration of both drugs and red light required makes PDT only useful on superficial cancer lesions. ${ }^{36}$ Surgery, on the other hand, can be curative for most of the skin tumour cases but side effects such as loss of function, scarring and on-going pain together with the morbidity and costs associated make it far from an ideal treatment. ${ }^{37}$ For skin infections, the use of topical and oral antibiotics are suggested in clinical guidelines. ${ }^{38}$ However, side effects after oral administration and reports addressing the rise in antibiotic resistance in skin infections shows the urgent need for alternative therapies. ${ }^{39,40}$

GnRs-mediated hyperthermia has been investigated as potential primary and adjunctive treatment for the aforementioned conditions since an increase in skin temperature over $43{ }^{\circ} \mathrm{C}$ can lead to bacterial and cancer cells death. ${ }^{2,3,41}$ Approaches where GnRs or other plasmonic materials (such as lanthanum hexaboride and graphene oxide), alone or in combination with antibiotic and antineoplastic drugs have been 
reported, showing that a temperature increase around $10-15{ }^{\circ} \mathrm{C}$ in in vivo studies was sufficient to achieve a photothermal effect. $^{7,10,42-45}$ However, topical, intratumoral and systemic toxicity of GnRs and other plasmonic materials has not been fully documented and literature is still controversial about the safety of these materials. ${ }^{21,22,25}$ Hence, until further information is gathered, research should focus on approaches that circumvent such administration routes.

Hereby we have reported a simple fabrication process of plasmonic devices, where a GnRs formulation was used to coat hydrogel-forming MN arrays and single needles. Although GnRs-coated single needles inserted deeper in the skin model and reached higher temperatures when exposed to laser irradiation, GnRs-coated MN arrays showed superiority in terms of heating capacity of a thick skin model. Upon insertion and irradiation of that prototype for only $15 \mathrm{~s}$, the underside of the skin model reached a temperature increase over $15{ }^{\circ} \mathrm{C}$, which confirms the feasibility of using hydrogelforming needles coated with GnRs for deep in skin hyperthermia applications.

\section{Conclusion}

To exploit the capacity of GnRs on inducing hyperthermia upon NIR laser irradiation, a novel and simple approach for the fabrication of plasmonic devices was followed. Hydrogelforming MN arrays and single needles were fabricated and then, coated with a GnRs-containing formulation. When heating capacity of a skin model was compared, GnRs-coated MN arrays heated up the skin in a higher degree than singles needles. Moreover, as the coating could be peeled off in one piece after insertion, irradiation and removal, chances of polymeric and plasmonic materials deposition in the skin are minimised. This technology circumvents current approaches where topical application and injection (intratumor, systemic) of GnRs are followed and, thereby, the uncertainty regarding GnRs metabolism, safety and toxicity. Future studies will now focus on testing GnRs-coated MN arrays efficacy as a treatment in murine models of skin bacterial infection and NMSC lesions. At this point, temperature increase and irradiation time needed to trigger a therapeutic response should be evaluated as well as GnRs release, assessing and quantifying whether plasmonic material is left in the skin after the device is removed.

\section{Author contributions}

A. C.-M., J. D.-R., A. S. C., R. F. D. contributed to the conceptualization of this study. A. C.-M., B. M., M. C. contributed to methodology. A. C.-M. and B. M. contributed to formal analysis. A. C.-M. contributed to the validation, investigation, data curation, writing-original draft preparation. J. D.-R., E. L., R. F. D. contributed to the writing-review and editing. S. E. J. B. and R. F. D. contributed to the supervision. R. F. D. contributed to resources, project administration and funding acquisition.

\section{Conflicts of interest}

There are no conflicts to declare.

\section{Acknowledgements}

The authors want to thank Luki Ahmadi Hari Wardoyo for his support designing the graphical abstract and also, the financial support of the SPaRK project at Queen's University Belfast, funded by the European Union's Horizon 2020 scheme under the Marie Skłodowska-Curie grant agreement No. 754507. This work was also supported by the Welcome Trust grant (WT094085MA), the Welcome Trust Biomedical Vacation Scholarship (216714/z/19/z) and the Engineering and Physical Sciences Research Council (EP/P034063/1).

\section{References}

1 P. Tiwari, K. Vig, V. Dennis and S. Singh, Nanomaterials, 2011, 1, 31-63.

2 T. Ibelli, S. Templeton and N. Levi-Polyachenko, Int. J. Hyperthermia, 2018, 34, 144-156.

3 S. K. Calderwood, J. R. Theriault and J. Gong, Int. J. Hyperthermia, 2005, 21, 713-716.

4 E. C. Dreaden, A. M. Alkilany, X. Huang, C. J. Murphy and M. A. El-Sayed, Chem. Soc. Rev., 2012, 41, 2740-2779.

5 V. P. Pattani, J. Shah, A. Atalis, A. Sharma and J. W. Tunnell, J. Nanopart. Res., 2015, 17, 20.

6 N. N. Mahmoud, A. A. Alhusban, J. I. Ali, A. G. Al-Bakri, R. Hamed and E. A. Khalil, Sci. Rep., 2019, 9, 1-15.

7 M. Liu, D. He, T. Yang, W. Liu, L. Mao, Y. Zhu, J. Wu, G. Luo and J. Deng, J. Nanobiotechnol., 2018, 16, 1-20.

8 M. S. Khan, M. L. Bhaisare, J. Gopal and H.-F. Wu, J. Ind. Eng. Chem., 2016, 36, 49-58.

9 F. H. Kao, N. Akhtar, C. C. Chen, H. Y. Chen, M. K. Thakur, Y. Y. Chen, C. L. Chen and S. Chattopadhyay, ACS Appl. Biol. Mater., 2019, 2, 533-543.

10 Y. H. Wang, S. P. Chen, A. H. Liao, Y. C. Yang, C. R. Lee, C. H. Wu, P. C. Wu, T. M. Liu, C. R. C. Wang and P. C. Li, Sci. Rep., 2014, 4, 1-8.

11 H. N. Green, S. D. Crockett, D. V. Martyshkin, K. P. Singh, W. E. Grizzle, E. L. Rosenthal and S. B. Mirov, Int. J. Nanomed., 2014, 9, 5093-5102.

12 E. B. Dickerson, E. C. Dreaden, X. Huang, I. H. El-Sayed, H. Chu, S. Pushpanketh, J. F. McDonald and M. A. El-Sayed, Cancer Lett., 2008, 269, 57-66.

13 A. G. Al-Bakri and N. N. Mahmoud, Molecules, 2019, 24, 2661.

14 M. A. Abdou Mohamed, V. Raeesi, P. V. Turner, A. Rebbapragada, K. Banks and W. C. W. Chan, Biomaterials, 2016, 97, 154-163.

15 W. Qi, J. Yan, H. Sun and H. Wang, Int. J. Nanomed., 2018, 13, 6235-6247.

16 A. Regiel-Futyra, M. Kus-Lis̈kiewicz, V. Sebastian, S. Irusta, M. Arruebo, G. Stochel and A. Kyzioł, ACS Appl. Mater. Interfaces, 2015, 7, 1087-1099. 
17 A. F. Moreira, C. F. Rodrigues, T. A. Jacinto, S. P. Miguel, E. C. Costa and I. J. Correia, Int. J. Pharm., 2020, 576, 118907.

18 Y. Hao, M. L. Dong, T. Y. Zhang, J. R. Peng, Y. P. Jia, Y. P. Cao and Z. Y. Qian, ACS Appl. Mater. Interfaces, 2017, 9, 15317-15327.

19 Y. Hao, Y. Chen, M. Lei, T. Zhang, Y. Cao, J. Peng, L. Chen and Z. Qian, Adv. Ther., 2018, 1, 1800008.

20 J. Wan, J. H. Wang, T. Liu, Z. Xie, X. F. Yu and W. Li, Sci. Rep., 2015, 5, 1-16.

21 S. Hashempour, S. Ghanbarzadeh, H. I. Maibach, M. Ghorbani and H. Hamishehkar, Ther. Delivery, 2019, 10, 383-396.

22 C. Grabinski, N. Schaeublin, A. Wijaya, H. D. Couto, S. H. Baxamusa, K. Hamad-schifferli and S. M. Hussain, ACS Nano, 2011, 5, 2870-2879.

23 N. M. Schaeublin, L. K. Braydich-Stolle, E. I. Maurer, K. Park, R. I. MacCuspie, A. R. M. N. Afrooz, R. A. Vaia, N. B. Saleh and S. M. Hussain, Langmuir, 2012, 28, 3248-3258.

24 S. Patibandla, Y. Zhang, A. M. Tohari, P. Gu, J. Reilly, Y. Chen and X. Shu, J. Appl. Toxicol., 2018, 38, 1153-1161.

25 M. van Pomeren, W. J. G. M. Peijnenburg, R. C. Vlieg, S. J. T. van Noort and M. G. Vijver, Nanotoxicology, 2019, 13, 558-571.

26 M. R. K. Ali, M. A. Rahman, Y. Wu, T. Han, X. Peng, M. A. Mackey, D. Wang, H. J. Shin, Z. G. Chen, H. Xiao, R. Wu, Y. Tang, D. M. Shin and M. A. El-Sayed, Proc. Natl. Acad. Sci. U. S. A., 2017, 114, E3110-E3118.

27 Á. Cárcamo-Martínez, J. Domínguez-Robles, B. Mallon, M. T. Raman, A. S. Cordeiro, S. E. J. Bell, E. Larrañeta and R. F. Donnelly, Nanomaterials, 2020, 10, 582.

28 X. Ye, L. Jin, H. Caglayan, J. Chen, G. Xing, C. Zheng, V. DoanNguyen, Y. Kang, N. Engheta, C. R. Kagan and C. B. Murray, ACS Nano, 2012, 6, 2804-2817.

29 Á. Cárcamo-Martínez, Q. K. Anjani, A. D. Permana, A. S. Cordeiro, E. Larrañeta and R. F. Donnelly, Int. J. Pharm., 2020, 2, 10048.
30 E. Larrañeta, J. Moore, E. M. Vicente-Pérez, P. GonzálezVázquez, R. Lutton, A. D. Woolfson and R. F. Donnelly, Int. J. Pharm., 2014, 472, 65-73.

31 E. Larrañeta, S. Stewart, S. J. Fallows, L. L. Birkhäuer, M. T. C. McCrudden, A. D. Woolfson and R. F. Donnelly, Int. J. Pharm., 2016, 497, 62-69.

32 E. D. SoRelle, O. Liba, Z. Hussain, M. Gambhir and A. de la Zerda, Langmuir, 2015, 31, 12339-12347.

33 T. R. Raj Singh, P. A. McCarron, A. D. Woolfson and R. F. Donnelly, Eur. Polym. J., 2009, 45, 1239-1249.

34 H. S. Gill and M. R. Prausnitz, J. Controlled Release, 2007, 117, 227-237.

35 A. L. Oldenburg, M. N. Hansen, D. A. Zweifel, A. Wei and S. A. Boppart, Opt. Express, 2006, 14, 6724.

36 C. Newlands, R. Currie, A. Memon, S. Whitaker and T. Woolford, J. Laryngol. Otol., 2016, 130, S125-S132.

37 L. Vallejo-Torres, S. Morris, J. M. Kinge, V. Poirier and J. Verne, J. Public Health, 2014, 36, 140-148.

38 D. L. Stevens, A. L. Bisno, H. F. Chambers, E. P. Dellinger, E. J. C. Goldstein, S. L. Gorbach, J. V. Hirschmann, S. L. Kaplan, J. G. Montoya and J. C. Wade, Clin. Infect. Dis., 2014, 59, e10-e52.

39 G. Perera and R. Hay, J. Eur. Acad. Dermatol. Venereol., 2005, 19, 531-545.

40 J. S. Lim, H. Park, S. Cho and H.-S. Yoon, Ann. Dermatol., 2018, 30, 186.

41 K. Ahmed, Y. Tabuchi and T. Kondo, Apoptosis, 2015, 20, 1411-1419.

42 M. C. Chen, Z. W. Lin and M. H. Ling, ACS Nano, 2016, 10, 93-101.

43 Y. Chen, Y. Yang, Y. Xian, P. Singh, J. Feng, S. Cui, A. Carrier, K. Oakes, T. Luan and X. Zhang, ACS Appl. Mater. Interfaces, 2020, 12, 352-360.

44 R. Mooney, E. Schena, P. Saccomandi, A. Zhumkhawala, K. Aboody and J. M. Berlin, Int. J. Hyperthermia, 2017, 33, 150-159.

45 R. Vankayala, Y. K. Huang, P. Kalluru, C. S. Chiang and K. C. Hwang, Small, 2014, 10, 1612-1622. 